Research Article

\title{
A Comparative Study between Air Bubble Tamponade Alone versus Air Bubble Tamponade with Internal Fluid Aspiration for Nonplanar Descemet's Membrane Detachment after Phacoemulsification
}

\author{
Amr A. Gab-Alla \\ Ophthalmology Department, Suez Canal University, Faculty of Medicine, Ismailia, Egypt \\ Correspondence should be addressed to Amr A. Gab-Alla; amrophth@hotmail.com
}

Received 22 March 2021; Revised 21 May 2021; Accepted 26 June 2021; Published 6 July 2021

Academic Editor: Achim Langenbucher

Copyright (C) 2021 Amr A. Gab-Alla. This is an open access article distributed under the Creative Commons Attribution License, which permits unrestricted use, distribution, and reproduction in any medium, provided the original work is properly cited.

\begin{abstract}
Purpose. To compare the efficacy of air bubble tamponade alone versus air bubble tamponade with internal fluid aspiration for nonplanar Descemet's membrane detachment after clear corneal incision phacoemulsification. Methods. This study is a prospective, intervention, comparative randomised clinical trial, conducted at a private eye centre, Ismailia, Egypt, from March 2019 to March 2020. It contained 30 eyes of 24 patients who had postphacoemulsification nonplanar Descemet's membrane detachment involving the periphery and the central area of the cornea ( $>50 \%$ of the cornea) with corneal oedema. The patients were divided into two groups: group A: patients with nonplanar DMD affecting the central cornea treated by air bubble tamponade only and group B: patients with nonplanar DMD affecting the central cornea treated by air bubble tamponade augmented by internal fluid aspiration. Trial Registration: This trial was registered at www.pactr.org and the identification number for the registry is PACTR202006612296119. Results. During the 12-month study period, this study included 30 eyes (24 patients) out of 1356 phaco surgeries with postphacoemulsification nonplanar Descemet's membrane detachment. Six patients had DMD in both eyes, eight patients had DMD in the right eye, and ten patients had DMD in the left eye. All patients have successful surgeries without complications. The calculated incidence rate for visually significant DMD was $2.2 \%$ per year. The mean \pm SD time interval from cataract surgery to the primary intervention was $4.2 \pm 1.1$ days. Descemet's membrane was attached in $56.25 \%$ of patients in group A ( 9 out of 16 eyes) and $92.6 \%$ of patients in group B (13 out of 14 eyes) with a minimum of one-month follow-up. Conclusion. Air descemetopexy combined with the internal fluid aspiration demonstrated to be more efficient than air descemetopexy only to treat Descemet's membrane detachment following phacoemulsification. It should be tried before planning other major surgeries in patients with severe Descemet's membrane detachment.
\end{abstract}

\section{Introduction}

Descemet's membrane detachment (DMD) is a severe postphacoemulsification complication that causes permanent corneal damage and decompensation. Even though developments in phacoemulsification innovation made it likely to do cataract surgery with microincision and get preferable postoperative results, a relatively high incidence of DMD has been recorded [1]. The incidence of clinically significant postphacoemulsification Descemet's membrane detachment (DMD) varies from 0.044 to $0.5 \%[2,3]$.
The potential causal factors include corneal dystrophy, shallow anterior chambers, complicated surgeries, dense nuclear cataracts, and accidental injection of viscoelastic substance or saline between Descemet's membrane and stroma [4-7]. Although rare cases of spontaneous reattachment of Descemet's membrane have been recorded [7], surgical interference to enhance reattachment is the standard method for most patients. Early intervention is important to avoid the wrinkling fibrosis and shrinkage of Descemet's membrane and to achieve good visual rehabilitation [8]. 
There is no gold standard for the treatment options of DMDs. Alternatives include observation, topical steroids, hyperosmotic agents, intracameral air or expandable gas injection, viscoelastic injection, transcorneal suturing, endothelial keratoplasty, and penetrating keratoplasty [9].

This study presents an alternative technique to treat postphacoemulsification Descemet's membrane detachment. It compares air bubble tamponade alone with air bubble tamponade with the internal fluid aspiration after clear corneal incision phacoemulsification.

\section{Methods}

This study is a prospective, intervention, comparative randomized clinical trial, conducted at a private eye centre, Ismailia, Egypt, from March 2019 to March 2020. It was registered at http://www.pactr.org and the identification number for the registry is PACTR202006612296119.

In this study, we followed the classification proposed by Mackool and Holtz [10], which categorised DMD into planar (separation $<1 \mathrm{~mm}$ ) and nonplanar (separation $>1 \mathrm{~mm}$ ) from the corneal stroma. They divided whether involvement is limited to the periphery of the cornea or involves the periphery and the central area of the cornea.

It contained 30 eyes of 24 patients who had postphacoemulsification nonplanar Descemet's membrane detachment involving the periphery and the central area of the cornea ( $>50 \%$ of the cornea) with corneal oedema.

Patients with nonplanar DMD affecting central cornea were divided randomly into two groups: group A treated by air bubble tamponade only and group B treated by air bubble tamponade augmented by the internal fluid aspiration.

Patients' eyes were randomly allocated to group A or group B using the simple randomization procedure. Allocation sequence was generated using the RAND () function in Microsoft Excel software (version 2016).

2.1. Inclusion Criteria. All the patients had coaxial phaco surgeries, through a $3 \mathrm{~mm}$ superior clear corneal incision with a single-use metal keratome and used Viscomed (hydroxypropyl methylcellulose 2.4\%; Albomed $\mathrm{GmbH}$, Germany) as an ophthalmic viscosurgical device. All patients had no previous intraocular surgery.

On the first postoperative day, patients with DMD were diagnosed on clinical grounds through slit-lamp examination. Anterior segment optical coherence tomography (Optovue RTVue, California, USA) was taken in all patients to confirm the diagnosis and determine the type and the extent of the detachment. Patients with nonplanar DMD involving $>50 \%$ of the cornea with central corneal involvement were included in the study.

2.2. Surgical Intervention. The interferences were completed under topical anaesthesia in the operation room to drain the predescemetic fluid joined with intracameral air bubble tamponade. The periocular area was sterilised with $10 \%$ povidone-iodine, and an anterior chamber paracentesis was performed in an area of attached DM. A 30-gauge hydrodissection cannula on a 2 cc syringe containing air was used to go below the detached DM, and the anterior chamber was filled with air. The paracentesis was fixed with a cotton wipe for about 1 minute to keep the air inside the anterior chamber and prevent it from getting away out. In group B patients, extra paracentesis was made with a 30gauge needle at the peripheral cornea to aspirate the internal fluid between the cornea and detached Descemet's membrane using the result of anterior segment optical coherence tomography (AS-OCT) as a guide. The needle stopped shortly after it penetrated the corneal stroma. The air was injected again into the anterior chamber through the initial paracentesis to push the predescemetic fluid out, along with aspiration with the syringe.

The anterior chamber was totally loaded up with air. Patients were kept in a supine position for 15 minutes, after which partial relief of air was done so that the anterior chamber was air-filled for almost two-thirds of its volume (Figure 1).

All processes followed the Declaration of Helsinki and adhered to CONSORT guidelines. It was approved by the Research Ethics Committee of the Faculty of Medicine, Suez Canal University. Written informed consent was obtained from the patients. The outcome was assessed as (1) anatomical success: reattachment of Descemet's membrane and (2) functional success: the resolution of corneal oedema.

The same surgeon (AAG) treated and followed all the patients in the two groups.

2.3. Postintervention Evaluation. The patients were advised to be in the supine position for the first day after the intervention. A combination of $0.3 \%$ tobramycin and $0.1 \%$ dexamethasone eye drops three times/day for the first week, decreased once/week, and antiglaucoma (brimonidine $0.1 \%$ ) eye drops three times/day was advised. Topical tropicamide $1 \%$ eye drops were given twice daily till complete resolution of the air bubble.

On the first postintervention day, the slit-lamp examination, IOP was noted. On follow-up at the first week and in the fourth week, the parameters noted were slit-lamp examination, IOP, BCVA, and AS-OCT to examine DM reattachment and complications of the intervention. If, after the primary intervention, there was significant persistent $\mathrm{DMD}$, reintervention was arranged. The time interval between the primary intervention and reinterventions was also recorded.

2.4. Statistical Analysis. All data handling and analysis were accomplished by Statistical Package for the Social Sciences (SPSS version 25.0; IBM Corporation, Armonk, NY, USA). Baseline characteristics of the study were presented as percentages and frequencies, or mean and standard deviations. The differences between frequencies in the two groups were compared through the chi-square test or Fisher's exact test (if $>20 \%$ of expected values were less than 5 ). The differences in quantitative measurements between the two groups were tested for statistical significance using the Mann-Whitney test. A Wilcoxon signed-rank test was used 


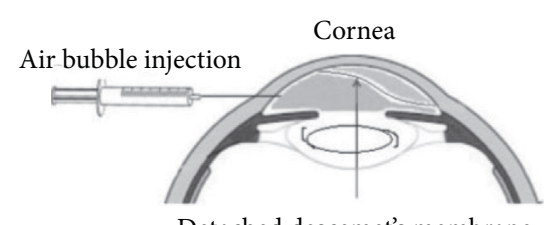

Detached descemet's membrane

(a)

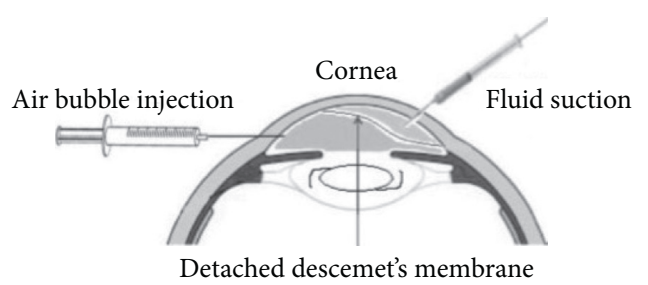

(b)

FIGURE 1: Schematic illustration of the surgical technique in both groups. (a) Group A. (b) Group B.

to compare the preintervention and postintervention measurements. A $p$ value $<0.05$ was considered statistically significant. The graphs were performed with GraphPad Prism (version 5.00 for Windows, GraphPad Software, La Jolla, California USA). Shapiro-Wilk's test was used to test for data normality.

\section{Results}

This study included 30 eyes (24 patients) out of 1356 phacoemulsification surgeries with postphacoemulsification nonplanar Descemet's membrane detachment (Figure 2). All cases were coaxial phaco surgeries, by $2.4 \mathrm{~mm}$ superior clear corneal incision fashioned with a disposable metal keratome and Optiflex (hydroxypropyl methylcellulose 2\%; Moss Vision Inc. Ltd., UK) as the viscoelastic device. Six patients had DMD in both eyes, eight patients had DMD in the right eye, and ten patients had DMD in the left eye. The mean \pm SD of the age of the patients was $62 \pm 2.1$ years and $61 \pm 3.2$ years in groups A and B, respectively. The male-tofemale ratio was $38.5: 61.5$ in group A and 45.5:54.5 in group B. All the patients have successful surgeries without complications with a minimum of 1-month follow-up. The calculated incidence rate per year (study time) for visually significant DMD was $2.2 \%$ (this rate does not include mild cases treated medically only). The patients were divided into two groups: group A included 16 eyes and group B included 14 eyes (Figure 1). The demographic data of both groups A and $\mathrm{B}$ are presented in Table 1.

3.1. DMD Site. DMD originated from main corneal incision was made in 23 eyes (76.7\%): 11 eyes (36.7\%) in group A and 12 eyes (40\%) in group B. Main and side incisions were made in 5 eyes (16.6\%): 3 eyes (10\%) in group A and 2 eyes (6.6\%) in group B. Side incisions were made in 2 eyes (6.7\%): 1 eye $(3.35 \%)$ in group A and 1 eye (3.35\%) in group B. Central cornea ( $>50 \%$ of the cornea) was involved in all cases.

3.2. Time of Resolution. The mean $\pm \mathrm{SD}$ of the time interval from phacoemulsification surgery to the primary intervention was $4.2 \pm 1.1$ days in group A and $4.3 \pm 0.9$ days in group B. After air descemetopexy, the DM was attached in 9 eyes (56.25\%) in group A and 13 eyes $(92.6 \%)$ in group B with a minimum of 1-month follow-up. There was a significant difference in the success rates between the two groups of patients $(p=0.039)$. The time of resolution was shorter in group B as compared to group A with a significant difference $\left(p=0.044^{*}\right)$ (Table 2). Six eyes of group A and one eye of group B needed secondary reinterventions. The mean \pm SD of the time interval for the reintervention was $9 \pm 2.1$ days. Patients who required reintervention were followed up for one month with successful reattachment of DM in $100 \%$ of all patients.

3.3. Visual Recovery. The mean $\pm \mathrm{SD}$ of the log MAR of the BCVA in both groups was improved from $0.95 \pm 0.65$ and $0.98 \pm 1.0$ to $0.47 \pm 2.1$ and $0.26 \pm 1.5$ in groups $A$ and $B$, respectively, with significant $p$ values $\left(0.042^{*}\right.$ and $\left.0.048^{*}\right)$ (Table 3 and Figure 3). Out of the 13 patients with attached DM in group A, 10 patients had improvement in BCVA 3 Snellen lines, and out of 11 patients in group B, 10 patients had improvement in BCVA 4 Snellen lines.

3.4. Corneal Thickness. The mean \pm SD of the corneal thickness was $766 \pm 18 \mu \mathrm{m}$ and $771 \pm 20 \mu \mathrm{m}$ improved to $554 \pm 22 \mu \mathrm{m}$ and $527 \pm 15 \mu \mathrm{m}$ in groups A and B, respectively $\left(p=0.001^{*}\right)$ at one month after the intervention (Table 3 and Figures 4 and 5).

3.5. Complications. All the patients had within normal IOP after the primary and the secondary reinterventions. No corneas decompensated during the follow-up of the study.

\section{Discussion}

Descemet's membrane detachment (DMD) is defined as separating Descemet's membrane from the posterior corneal stroma. Mild DMD is visually insignificant, commonly overlooked (or if diagnosed), which is managed with topical hyperosmotic agents. More likely, they get spontaneously reattached after some time [11].

The main corneal incision possibly experiences forceful fluid currents through a snug-fitting between the phaco machine's probe and the clear corneal incision, therefore a higher risk of accidental DM stripping, mainly at this site [12].

Weng et al. [13] reported in this case study that drainage of predescemetic fluid joined with intracameral air bubble tamponade was another surgical alternative for managing a severe case of DMD. This study is the first yet reported series of postphacoemulsification nonplanar DMD, with almost $92.6 \%$ of the patients attaining successful reattachment of 


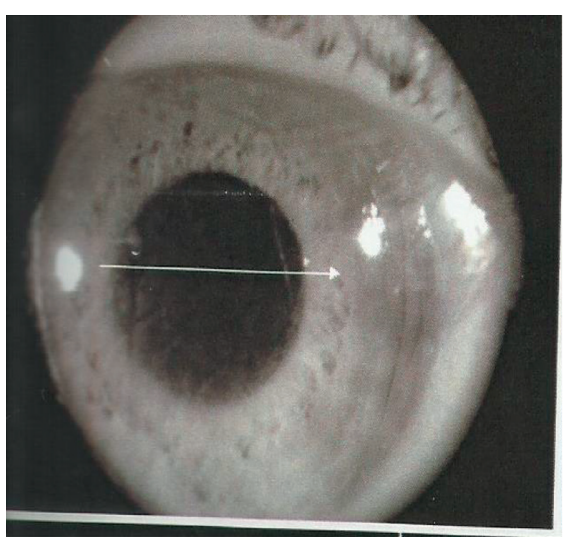

(a)

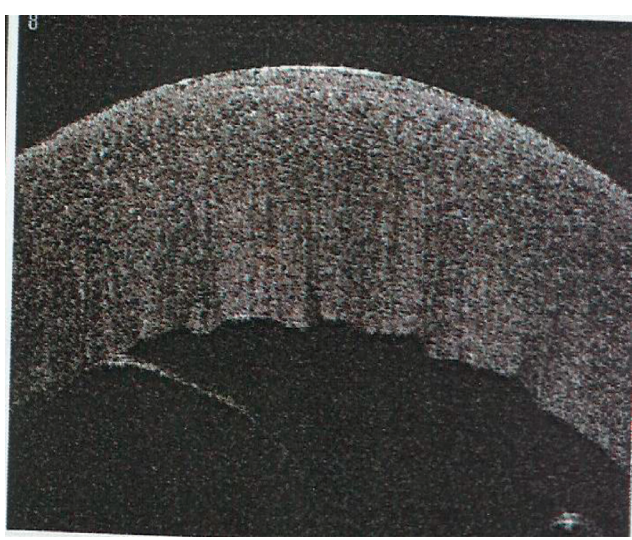

(b)

Figure 2: Preintervention nonplanar Descemet's membrane detachment affecting the central cornea with severe corneal oedema.

TABle 1: Preoperative demographic data for both groups.

\begin{tabular}{|c|c|c|c|}
\hline Demographic data & Group A ( $n=16$ eyes, 13 ptns) & Group B ( $n=14$ eyes, 11 ptns) & $p$ value \\
\hline \multicolumn{4}{|l|}{ Eye } \\
\hline Rt $(n)$ & 6 & 8 & \multirow{2}{*}{0.290} \\
\hline Lt $(n)$ & 10 & 6 & \\
\hline \multicolumn{4}{|l|}{ Bilaterality } \\
\hline Bilateral $(n)$ & 4 & 2 & \multirow{2}{*}{0.640} \\
\hline Unilateral $(n)$ & 8 & 10 & \\
\hline \multicolumn{4}{|l|}{ Sex } \\
\hline Male, $n(\%)$ & $5(38.5 \%)$ & $5(45.5 \%)$ & \multirow{2}{*}{0.735} \\
\hline Female, $n(\%)$ & $8(61.5 \%)$ & $6(54.5 \%)$ & \\
\hline \multicolumn{4}{|l|}{ Age (years) } \\
\hline Mean \pm SD & $62 \pm 2.1$ & $61 \pm 3.2$ & 0.426 \\
\hline \multicolumn{4}{|l|}{$B C V A(\log M A R)$} \\
\hline Mean \pm SD & $0.95 \pm 0.65$ & $0.98 \pm 1.0$ & 0.982 \\
\hline
\end{tabular}

Notes. Group A: patients with nonplanar DMD affecting the central cornea treated by air bubble tamponade only; group B: patients with nonplanar DMD affecting the central cornea treated by air bubble tamponade with internal fluid aspiration. $n$ : number; SD: standard deviation; ptns: patients.

TABLE 2: Postoperative success rates of both groups.

\begin{tabular}{|c|c|c|c|}
\hline Parameter & Group $A, n=16$ & Group $\mathrm{B}, n=14$ & $p$ value \\
\hline Time of intervention: mean \pm SD (days) & $4.1 \pm 1.1$ & $4.3 \pm 0.9$ & 0.623 \\
\hline Time of resolution: mean \pm SD (days) & $1.9 \pm 1.2$ & $1.1 \pm 0.8$ & $0.044^{*}$ \\
\hline Reattachment: $n(\%)$ & $9(56.25 \%)$ & $13(92.6 \%)$ & $0.039^{*}$ \\
\hline
\end{tabular}

Notes. Group A: patients with nonplanar DMD affecting the central cornea treated by air bubble tamponade only; group B: patients with nonplanar DMD affecting the central cornea treated by air bubble tamponade with internal fluid aspiration. SD: standard deviation; $n$ : number; BCVA: best-corrected visual acuity. ${ }^{*}$ Statistically significant $(p$ value $<0.05)$.

TABle 3: Pre- and postintervention log MAR of BCVA and corneal thickness in both groups at one month after the intervention.

\begin{tabular}{|c|c|c|c|c|}
\hline Measurement & Time & Group A & Group B & $p 1$ value \\
\hline BCVA in log MAR & $\begin{array}{l}\text { Preintervention: mean } \pm \mathrm{SD} \\
\text { Postintervention: mean } \pm \mathrm{SD} \\
\quad p^{2} \text { value }\end{array}$ & $\begin{array}{c}0.95 \pm 0.65 \\
0.47 \pm 0.6 \\
0.042^{*}\end{array}$ & $\begin{array}{c}0.98 \pm 1.0 \\
0.26 \pm 0.8 \\
0.048^{*}\end{array}$ & $\begin{array}{l}0.965 \\
0.520\end{array}$ \\
\hline Corneal thickness $(\mu \mathrm{m})$ AS-OCT & $\begin{array}{l}\text { Preintervention: mean } \pm \mathrm{SD} \\
\text { Postintervention: mean } \pm \mathrm{SD} \\
\quad p 3 \text { value }\end{array}$ & $\begin{array}{l}766 \pm 18 \\
554 \pm 22 \\
<0.001^{*}\end{array}$ & $\begin{array}{l}771 \pm 20 \\
527 \pm 15 \\
<0.001^{*}\end{array}$ & $\begin{array}{c}0.512 \\
<0.001^{*}\end{array}$ \\
\hline
\end{tabular}

Notes. Group A: patients with nonplanar DMD affecting the central cornea treated by air bubble tamponade only; group B: patients with nonplanar DMD affecting the central cornea treated by air bubble tamponade with internal fluid aspiration. SD: standard deviation; BCVA: best-corrected visual acuity; ASOCT: anterior segment optical coherence tomography. ${ }^{*}$ Statistically significant. 


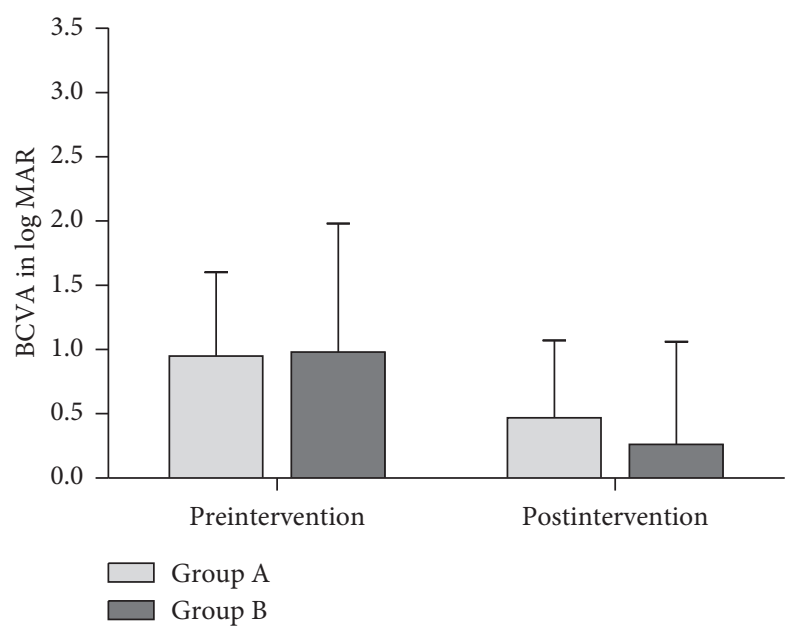

FIGURE 3: Pre- and postintervention mean of the log MAR of BCVA at one month in both groups.

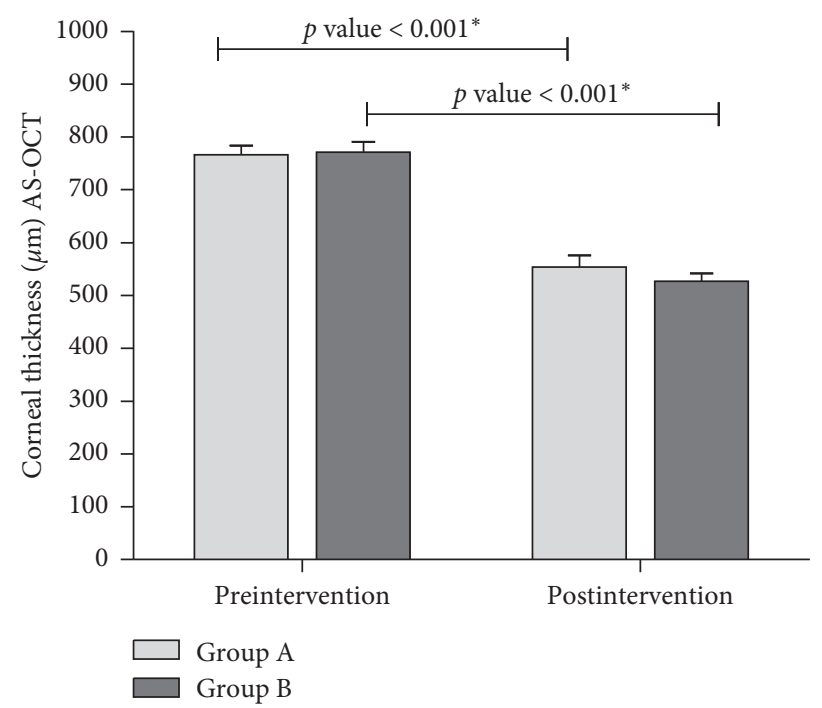

Figure 4: Pre- and postintervention mean of the central corneal thickness (CCT) of both groups at one month after the intervention.

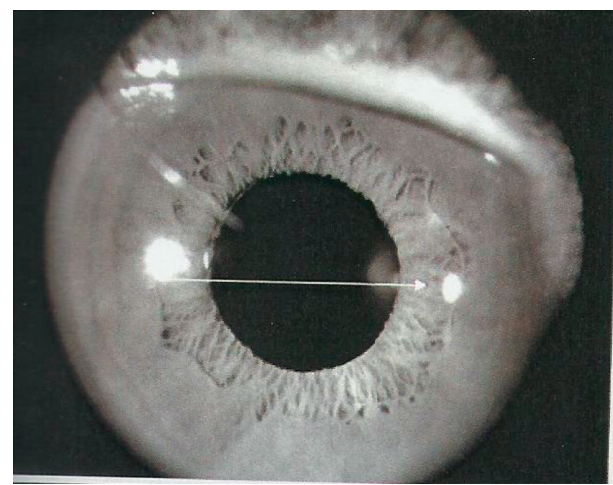

(a)

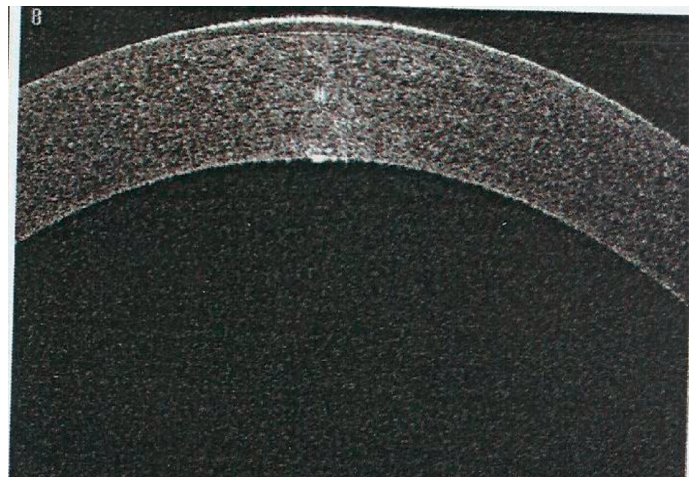

(b)

FIGURE 5: Postintervention with complete Descemet's membrane reattachment and resolution of corneal oedema. 
DM with air bubble tamponade augmented with internal fluid aspiration versus $56.25 \%$ in patients with only air tamponade. Also, the time of resolution was faster in group $\mathrm{B}$ versus group A with a significant difference $\left(p=0.044^{*}\right)$. Nonplanar DMD, to some extent, resembles a serous retinal detachment in appearance. When the surgeon is doing stromal hydration after a successful phacoemulsification, it is normal to see this. Holding the cannula close to the posterior stroma may cause the fluid wave to spread between the stroma and Descemet's membrane causing DMD, which is basically fluid stuck under Descemet's membrane as a slightly convex (fluid pocket) into the anterior chamber (Figure 2). An exit must be created for the fluid to evacuate completely and the detachment to fully decompress with a tamponade agent to allow reattachment. The injected air in the anterior chamber raises the detached Descemet's membrane up to adhere to the stroma of the cornea. At the same time, the drainage of predescemetic fluid externally by syringe aspiration (analogous to internal tamponade and subretinal fluid drainage in retinal detachment) helps Descemet's membrane stick to the collagen of the cornea and prevents the detachment from more expansion. It is simple to understand that air bubble alone will not completely work. This allows the membrane to adhere faster and corneal oedema to improve earlier and visual acuity improvement with a minimally invasive surgical manipulation to the cornea. This is considered an important finding, suggesting that air tamponade with internal fluid aspiration is worth trying, especially in severe cases of DMD. This helps to prevent major surgeries such as penetrating keratoplasty.

The calculated incidence rate for visually significant DMD in this study was $2.2 \%$ per year (30 out of 1356 phacoemulsification surgeries). This is a relatively high incidence rate compared to other studies $[2,5,8,14,15]$. This could be explained by the excessive wound hydration, hardness of the cataract, hot climate, or late cataract surgeries for economic reasons. Genetic factors (preoperative corneal dystrophies) may play a role in this high rate. Relevant to that, Anderson [16] reported a 5\% incidence rate of DMD, and Jaramillo et al. [17] reported a 7.4\% incidence rate of DMD. Some authors reported up to $43 \%$ incidence rate of DMD [18].

Air is usually preferred as a tamponading agent for many causes, including its lower cost, shorter absorption time, and low risk of endothelial toxicity or pupillary block than with other long-standing gases including 14\% perfluoropropane and $20 \%$ sulphur hexafluoride $[19,20]$. Tamponading with viscoelastic agents has been reported in some previous studies $[21,22]$ as a treatment option for DMD, but this method has some limitations because of the high risk of elevating the intraocular pressure and the need for continuous follow-up and monitoring [3].

One limitation of this study was underestimation of the true incidence of DMD since only moderate and severe nonplanar DMD were included, while mild cases were not, which are visually insignificant (usually missed or are managed with topical hyperosmotic agents). Second, the relationship between the grade of the cataract and the incidence of DMD was not assessed. Last, whether the success of surgeries is affected to some extent by the state of the endothelium which needs further study on the preoperative assessment by the specular microscopy.

In conclusion, air descemetopexy combined with internal fluid aspiration demonstrated to be more efficient than air descemetopexy only to treat Descemet's membrane detachment following phacoemulsification. It should be tried before planning other major surgeries in patients with severe Descemet's membrane detachment.

\section{Data Availability}

The datasets used and/or analysed during the current study are available from the corresponding author on reasonable request.

\section{Conflicts of Interest}

The author declares that he has no conflicts of interest.

\section{Supplementary Materials}

CONSORT 2010 checklist of information to include when reporting a randomised trial. (Supplementary Materials)

\section{References}

[1] B. Cheng, Y. Liu, Y. Liu, B. B. Xie, L. Xi, and Y. Yang, "Early changes in morphology and intraocular pressure by size of clear corneal incision," Cornea, vol. 30, no. 6, pp. 634-640, 2011.

[2] S.-E. Ti, S.-P. Chee, D. T. H. Tan, Y.-N. Yang, and S. L. Shuang, "Descemet membrane detachment after phacoemulsification surgery," Cornea, vol. 32, no. 4, pp. 454-459, 2013.

[3] V. W. S. Chow, T. Agarwal, R. B. Vajpayee, and V. Jhanji, "Update on diagnosis and management of Descemet's membrane detachment," Current Opinion in Ophthalmology, vol. 24, no. 4, pp. 356-361, 2013.

[4] S. Datar, A. Kelkar, A. K. Jain et al., "Repeat Descemetopexy after Descemet's membrane detachment following phacoemulsification," Case Reports in Ophthalmology, vol. 5, no. 2, pp. 203-206, 2014.

[5] D. A. Kumar, A. Agarwal, S. Sivanganam, and R. Chandrasekar, "Height-, extent-, length-, and pupil-based (HELP) algorithm to manage post-phacoemulsification Descemet membrane detachment," Journal of Cataract and Refractive Surgery, vol. 41, no. 9, pp. 1945-1953, 2015.

[6] N. Sharma, P. Bandivadekar, T. Agarwal, R. Shah, and J. S. Titiyal, "Incision-site Descemet membrane detachment during and after phacoemulsification," Eye \& Contact Lens: Science \& Clinical Practice, vol. 41, no. 5, pp. 273-276, 2015.

[7] D. Singhal, P. Sahay, S. Goel, M. I. Asif, P. K. Maharana, and N. Sharma, "Descemet membrane detachment," Survey of Ophthalmology, vol. 65, no. 3, pp. 279-293, 2020.

[8] J. Francois, J.-C. Vermion, G. Hayek et al., "Management of large central Descemet membrane detachment (DMD) after cataract surgery: case report and literature review," Journal Français d'Ophtalmologie, vol. 42, no. 6, pp. e271-e278, 2019.

[9] S. Jacob, A. Agarwal, A. Agarwal, and S. Ai, "Recognizing and managing bullous Descemet detachment secondary to accidental hydroseparation during phacoemulsification/cataract surgery by relaxing descemetotomy," Cornea, vol. 37, no. 3, pp. 394-399, 2018. 
[10] M. Das, M. Begum Shaik, N. Radhakrishnan, and V. N. Prajna, "Descemet membrane suturing for large Descemet membrane detachment after cataract surgery," Cornea, vol. 39, no. 1, pp. 52-55, 2020.

[11] P. Guo, Y. Pan, Y. Zhang et al., "Study on the classification of Descemet membrane detachment after cataract surgery with AS-OCT," International Journal of Medical Sciences, vol. 15, no. 11, pp. 1092-1097, 2018.

[12] R. J. Mackool and S. J. Holtz, "Descemet membrane detachment," Archives of Ophthalmology, vol. 95, no. 3, pp. 459-463, 1977.

[13] Y. Weng, Y.-P. Ren, L. Zhang, X.-D. Huang, and X.-C. Shen$\mathrm{Tu}$, "An alternative technique for Descemet's membrane detachment following phacoemulsification: case report and review of literature," BMC Ophthalmology, vol. 17, no. 1, p. 109, 2017.

[14] S. Jacob, A. Agarwal, P. Chaudhry, S. Narasimhan, and V. N. Chaudhry, "A new clinico-tomographic classification and management algorithm for Descemet's membrane detachment," Contact Lens and Anterior Eye, vol. 38, no. 5, pp. 327-333, 2015.

[15] A. Odyappan, N. Shivananda, S. Ramakrishnaan, T. Krishnaan, S. Nachiappan, and S. Krishnamurthy, "A retrospective study on the incidence of post-cataract surgery Descemet's membrane detachment outcome of air descemetopexy," British Journal of Ophthalmology, vol. 102, no. 2, pp. 182-186, 2018.

[16] J. C. Anderson, "Gonioscopy in no-stitch cataract incisions," Journal of Cataract and Refractive Surgery, vol. 19, no. 5, pp. 620-621, 1993.

[17] A. Jaramillo, J. Foreman, and R. S. Ayyala, "Descemet membrane detachment after canaloplasty," Journal of Glaucoma, vol. 23, no. 6, pp. 351-354, 2014.

[18] C. A. Benatti, J. Z. Tsao, and N. A. Afshari, "Descemet membrane detachment during cataract surgery," Current Opinion in Ophthalmology, vol. 28, no. 1, pp. 35-41, 2017.

[19] R. Jain, S. I. Murthy, S. Basu, M. H. Ali, and V. S. Sangwan, "Anatomic and visual outcomes of descemetopexy in postcataract surgery Descemet's membrane detachment," Ophthalmology, vol. 120, no. 7, pp. 1366-1372, 2013.

[20] A. Sharma, S. K. Singh, P. L. Bhutia, and R. Pant, "Perfluoropropane (C3F8) injection for Descemet's membrane detachment in cataract surgery," Nepalese Journal of Ophthalmology, vol. 7, no. 1, pp. 74-78, 2015.

[21] P. B. Donzis, Z. A. Karcioglu, and M. S. Insler, "Sodium hyaluronate (Healon) in the surgical repair of Descemet's membrane detachment," Ophthalmic Surgery, Lasers and Imaging Retina, vol. 17, no. 11, pp. 735-737, 1986.

[22] K. Sonmez, P. Y. Ozcan, and A. G. Altintas, "Surgical repair of scrolled Descemet's membrane detachment with intracameral injection of $1.8 \%$ sodium hyaluronate," International Ophthalmology, vol. 31, no. 5, pp. 421-423, 2011. 